\title{
Electro-Thermal Analysis of Submicron Si MOSFET with Zoned Mesh Based on Semiconductor Physics Theory*
}

\author{
Tomoyuki HATAKEYAMA $^{* *, * * *}$,Kazuyoshi FUSHINOBU ${ }^{* *}$ and Ken OKAZAKI ${ }^{* *}$ \\ ** Department of Mechanical and Control Engineering, Tokyo Institute of Technology, \\ 2-12-1 O-okayama, Meguro-ku, Tokyo, 152-8552, Japan \\ E-mail : hatake@stu.mech.titech.ac.jp \\ *** JSPS Research Fellow
}

\begin{abstract}
The results of electro - thermal analysis, which is widely known as hydrodynamic model, are strongly dependent on the mesh size of model. However, the theory and method of accurate mesh size have not been investigated. In this research, we focus on submicron Si MOSFET and show the mesh zoning method for electro - thermal analysis. In the previous study, the authors proposed the mesh zoning method for vertical direction of Si MOSFET, i.e. the direction from the gate oxide to the bottom surface of MOSFET. The mesh zoning method was derived from the theory of the semiconductor physics. In this paper, the mesh zoning method for the lateral direction, i.e. the direction from the source electrode to the drain electrode, is considered. The calculation results show the most important point of mesh zoning for lateral direction is pinch-off point in the electron channel of MOSFET. Further, in the case that the fine meshes are used around the pinch-off point and wider meshes are used for other region, the results show good agreement with the results of the fine mesh model.
\end{abstract}

Key words: Electro-Thermal Analysis, Si MOSFET, Debye Length, Mesh Zoning

\section{Introduction}

The process size of semiconductor devices has reached the range of sub-100 nm length scale. The report of International Technology Roadmap for Semiconductors (ITRS) '05 (1) has originally predicted that $65 \mathrm{~nm}$ process would be realized in 2007. LSI (Large Scale Integration) is the most important part in the electrical devices, and mainly consists of $\mathrm{Si}$ MOSFETs (Metal-Oxide-Semiconductor Field Effect Transistors). The development of manufacturing process of semiconductor devices leads to the miniaturizing of Si MOSFETs. This means the increase of the MOSFETs density in LSI. Due to the increase of the heat dissipating devices in the LSIs, thermal problem will be a major task for packaging community. Due to the decrease of the device feature size, new type of thermal problem, submicron scale local hot spot, may become important. The local hot spot is a high temperature region within the heat dissipating devices, and is already important for semiconductor devices with lower thermal conductivities. Because of decrease in device feature size and multilayer structures, it may become important for future silicon devices. The key idea to tackle this problem is to precisely predict the heat dissipation distribution in the devices, and for this purpose, one must consider the behavior of the electrical as well as the thermal characteristics. ${ }^{(2)}$ To consider the behavior of the electrical and the thermal characteristics of semiconductor devices, electro - thermal analysis, which is widely known

*Received 18 Sep., 2007 (No. 07-0564) [DOI: 10.1299/jtst.3.45] 
as hydrodynamic model, is attractive ${ }^{(3)(4)}$ and widely used technique. However, the calculation accuracy of this analysis strongly depends on the mesh size of the calculation model. Especially, considering the inversion layer (the channel region) of Si MOSFET, the carrier density has abrupt change and the mesh size should be given careful consideration because the device characteristics strongly depend on the inversion carrier density. However, the theory and method of accurate mesh size has not been investigated. We proposed the accurate mesh size and mesh zoning method for the vertical direction, i.e. the direction from the gate oxide to the bottom surface of MOSFET, for precise calculation of device behavior of submicron Si MOSFET ${ }^{(5)}$. In that work, we especially focused on the vertical direction because the calculation results were strongly dependent on the mesh size for the vertical direction. Since our mesh zoning method was derived from the semiconductor physics theory, our method can be easily applied to any calculations of MOSFET. However, in our previous work, the accurate mesh size and mesh zoning method for the lateral direction, i.e. the direction from the source electrode to the drain electrode, were not considered. (Note: In previous our study ${ }^{(5)}$, the calculation results were not strongly dependent on the mesh size for the lateral direction. The difference of calculated drain current between the fine mesh model, where the mesh size for the lateral direction is 1 $\mathrm{nm}$, and the large mesh model, where the size for the lateral direction is $10 \mathrm{~nm}$, was less than $3 \%$. Therefore, we didn't care the mesh size for the lateral direction. However, in this study, the difference of calculated drain current between the fine mesh model and the large mesh model is larger than that of the previous study. The difference between this study and the previous study is the carrier generation/recombination model. In the previous study, the carrier generation/recombination was dependent on the carrier temperature. On the other hand, the carrier generation/recombination of this study depends on the electric field. That is the reason why we should take care the mesh size for the lateral direction. In this study case, the mesh zoning method for the vertical direction proposed by us is still suitable. The calculation results with zoned mesh for the vertical direction show good agreement with that with fine mesh, where the mesh size for the vertical direction is $1 \mathrm{~nm}$. We already checked that the difference of calculated drain current between those models was about $5 \%$. Therefore, the mesh zoning method proposed by us is applied for vertical direction and we investigate the suitable mesh size for the lateral direction.)

In this paper, we briefly show the procedure of our mesh zoning method for the vertical direction and we discuss the position in where the mesh size for the lateral direction should be given careful consideration. Further, the results of several models with several kind of zoned mesh are compared and we propose the mesh zoning method for the lateral direction of MOSFET.

\section{Nomenclature}

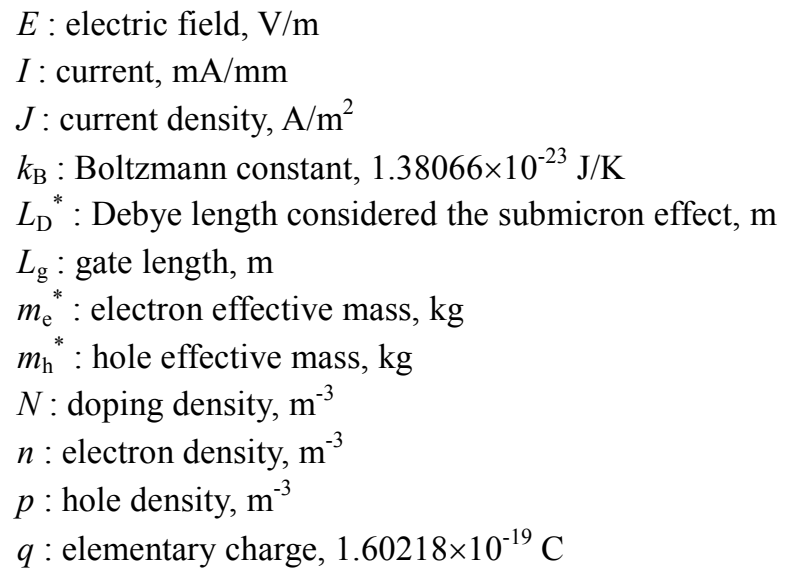


$R$ : net recombination rate $1 /\left(\mathrm{m}^{3} \cdot \mathrm{s}\right)$

$T$ : temperature, $\mathrm{K}$

$V$ : voltage, $\mathrm{V}$

$v:$ velocity, $\mathrm{m} / \mathrm{s}$

$W$ : carrier energy, $\mathrm{J} / \mathrm{m}^{3}$

\section{Greek symbols}

$\varepsilon_{\mathrm{Si}}:$ permittivity of silicon

$\varepsilon_{\mathrm{ax}}$ : permittivity of oxide $\left(\mathrm{SiO}_{2}\right)$

$\kappa$ : thermal conductivity, $\mathrm{W} /(\mathrm{m} \cdot \mathrm{K})$

$\mu:$ mobility, $\mathrm{m}^{2} /(\mathrm{V} \cdot \mathrm{s})$

$\tau$ : relaxation time, $\mathrm{s}$

$\phi$ : electrical potential, $\mathrm{V}$

\section{Subscripts}
A : acceptor
$\mathrm{D}:$ donor or drain
e : electron
e-L : electron to crystal lattice
$\mathrm{G}$ : gate
$\mathrm{h}$ : hole
h-L : hole to crystal lattice
$\mathrm{i}$ : intrinsic
$\mathrm{L}:$ crystal lattice (silicon in this literature)
$\mathrm{m}$ : momentum
ox : oxide
$\mathrm{S}$ : source
$\mathrm{Si}$ : silicon

\section{Governing Equations}

To analyze the thermal and electrical behavior of the devices, it is important to consider the case when the carrier temperatures are not equivalent to the lattice temperature. To consider such non-equilibrium state of temperature, electro-thermal analysis, which is known as hydrodynamic model, is a widely used technique. In this section, we show the details of electro-thermal analysis.

The set of governing equations of electro-thermal analysis is composed of Poisson equation, the continuity equations for electrons and holes, the momentum conservation equations for electrons and holes and the energy conservation equations for electrons, holes and the crystal lattice. The details of these equations are shown below. ${ }^{(3) \sim(6)}$

$$
\begin{aligned}
& \nabla^{2} \phi=-\frac{q}{\varepsilon_{S i}}\left(N_{D}-n-N_{A}+p\right) \\
& \nabla \cdot\left(n \mathbf{v}_{e}\right)=-R \\
& \nabla \cdot\left(p \mathbf{v}_{h}\right)=-R \\
& -q n \nabla \phi+\nabla\left(n k_{B} T_{e}\right)=-\frac{n m_{e}^{*} \mathbf{v}_{e}}{\tau_{m e}} \\
& q p \nabla \phi+\nabla\left(p k_{B} T_{h}\right)=-\frac{p m_{h}^{*} \mathbf{v}_{h}}{\tau_{m h}}
\end{aligned}
$$




$$
\begin{aligned}
& \nabla \cdot\left(\mathbf{v}_{e} W_{e}\right)-q n \mathbf{v}_{e} \nabla \phi+\nabla \cdot\left(\mathbf{v}_{e} n k_{B} T_{e}\right) \\
& -\nabla \cdot\left(\kappa_{e} \nabla T_{e}\right)=-\frac{W_{e}-W_{e 0}}{\tau_{e-L}} \\
& \nabla \cdot\left(\mathbf{v}_{h} W_{h}\right)+q p \mathbf{v}_{h} \nabla \phi+\nabla \cdot\left(\mathbf{v}_{h} p k_{B} T_{h}\right) \\
& -\nabla \cdot\left(\kappa_{h} \nabla T_{h}\right)=-\frac{W_{h}-W_{h 0}}{\tau_{h-L}} \\
& -\nabla \cdot\left(\kappa_{L} \nabla T_{L}\right)=\frac{W_{e}-W_{e 0}}{\tau_{e-L}}+\frac{W_{h}-W_{h 0}}{\tau_{h-L}}
\end{aligned}
$$

Equation (1) is the Poisson equation. Electrical potential, $\phi$, is calculated from this equation. Equations (2) and (3) are the continuity equation for the electrons and for the holes respectively. $R$ represents net recombination rate. In this work, Shockley-Read-Hall (SRH) recombination and impact ionization are considered. SRH recombination is expressed as below. ${ }^{(7)}$

$$
\begin{aligned}
R_{S R H} & =\frac{n p-n_{i}^{2}}{\tau_{S R H-h}\left(n+n_{i}\right)+\tau_{S R H-e}\left(p+n_{i}\right)} \\
\tau_{S R H-e} & =\frac{\tau_{e 0}}{1+\frac{N_{D}+N_{A}}{N_{e}^{\text {ref }}}} \\
\tau_{S R H-h} & =\frac{\tau_{h 0}}{1+\frac{N_{D}+N_{A}}{N_{h}^{r e f}}}
\end{aligned}
$$
$\left[\mathrm{m}^{-3}\right]$.

where $\tau_{\mathrm{e} 0}=3.95 \times 10^{-4}[\mathrm{~s}], N_{\mathrm{e}}^{\text {ref }}=7.1 \times 10^{21}\left[\mathrm{~m}^{-3}\right], \tau_{\mathrm{h} 0}=3.53 \times 10^{-5}[\mathrm{~s}]$ and $N_{\mathrm{h}}{ }^{\text {ref }}=7.1 \times 10^{21}$

In this work, the electric field dependence model of impact ionization is applied and impact ionization model is given as

$$
\begin{aligned}
& R_{I I}=-\alpha_{e} \cdot \frac{\left|\vec{J}_{e}\right|}{q}-\alpha_{h} \cdot \frac{\left|\vec{J}_{h}\right|}{q} \\
& \alpha_{e}=\alpha_{e}^{\infty} \cdot \exp \left(-E_{e}^{c r i t} / E\right) \\
& \alpha_{h}=\alpha_{h}^{\infty} \cdot \exp \left(-E_{h}^{c r i t} / E\right)
\end{aligned}
$$

where $\alpha_{\mathrm{e}}^{\infty}=7.03 \times 10^{7}\left[\mathrm{~m}^{-1}\right], E_{\mathrm{e}}{ }^{\text {crit }}=1.231 \times 10^{8}\left[\mathrm{Vm}^{-1}\right], \alpha_{\mathrm{h}}{ }^{\infty}=1.582 \times 10^{8}\left[\mathrm{Vm}^{-1}\right]$ and $E_{\mathrm{h}}{ }^{\text {crit }}$ $=2.036 \times 10^{8}\left[\mathrm{Vm}^{-1}\right]$ for $E \leq 4 \times 10^{7}, \alpha_{\mathrm{h}}^{\infty}=6.71 \times 10^{7}\left[\mathrm{~m}^{-1}\right]$ and $E_{\mathrm{h}}{ }^{\text {crit }}=1.693 \times 10^{8}\left[\mathrm{Vm}^{-1}\right]$ for $E$ $>4 \times 10^{7}\left[\mathrm{Vm}^{-1}\right]{ }^{(7)}$

Equations (4) and (5) are the momentum conservation equations for the electrons and the holes respectively. From Eq. (4), the electron velocity is defined, with the electron velocity is given as

$$
\mathbf{v}_{e}=\mu_{e} \nabla \phi-\frac{\mu_{e}}{q n} \nabla\left(n k_{B} T_{e}\right)
$$

where

$\mu_{e}=\frac{q \tau_{m e}}{m_{e}^{*}}$

The hole velocity is also obtained from Eq. (5), with the hole velocity is given as

$\mathbf{v}_{h}=-\mu_{h} \nabla \phi-\frac{\mu_{h}}{q p} \nabla\left(p k_{B} T_{h}\right)$

where

$\mu_{h}=\frac{q \tau_{m h}}{m_{h}^{*}}$

Therefore, electron density, $n$, and hole density, $p$, are calculated from Eqs. (2) and (3).

The electron mobility, $\mu_{\mathrm{e}}$, and the hole mobility, $\mu_{\mathrm{h}}$, depend on the scattering with phonon, impurities, surface et cetera. In this research, the scattering with phonon and impurities are considered. ${ }^{(7)}$ The mobility model is given as 


$$
\begin{aligned}
\mu_{e}^{I} & =90 \times 10^{4} \cdot\left(1+\frac{2 \times 10^{24}}{C I}\left(\frac{T_{L}}{300}\right)\right) \\
\mu_{h}^{I} & =45 \times 10^{4} \cdot\left(1+\frac{1.2 \times 10^{24}}{C I}\left(\frac{T_{L}}{300}\right)\right) \\
\mu_{e}^{L} & =\frac{1}{\frac{1}{0.4195\left(\frac{T_{L}}{300}\right)^{-1.5}}+\frac{0.2153\left(\frac{T_{L}}{300}\right)^{-3.13}}{1}} \\
\mu_{h}^{L}= & \frac{1}{0.2502\left(\frac{T_{L}}{300}\right)^{-1.5}+\frac{1}{0.0591\left(\frac{T_{L}}{300}\right)^{-3.25}}} \\
\mu_{e, h}^{L I}= & \left(\frac{1}{\mu_{e, h}^{I}}+\frac{1}{\mu_{e, h}^{L}}\right)^{-1}
\end{aligned}
$$

where $C I$ is impurity density.

For high electric field, the carrier mobility strongly depends on the electric field and the carrier velocity becomes saturated. This effect is given as

$$
\mu_{e, h}^{L I E}=\frac{\mu_{e, h}^{L I}}{\left.\sqrt{1+\left(\mu_{e, h}^{L I}\right)^{2}\left(\frac{E}{\frac{\mu_{e, h}^{L} \cdot A_{e, h}}{\mu_{e, h}^{L} \cdot A_{e, h}}+F_{e, h}}+\left(\frac{E}{\mu_{e, h}^{L} \cdot B_{e, h}}\right)^{2}\right.}\right)}
$$

where $A_{e}=3.5 \times 10^{5}[\mathrm{~V} / \mathrm{m}], F_{e}=8.8, B_{e}=7.4 \times 10^{5}[\mathrm{~V} / \mathrm{m}], A_{\mathrm{h}}=6.1 \times 10^{5}[\mathrm{~V} / \mathrm{m}], F_{\mathrm{h}}=1.6$ and $B_{\mathrm{h}}=2.5 \times 10^{6}[\mathrm{~V} / \mathrm{m}]$.

Equation (6) is the energy conservation equation for electrons. The electron temperature, $T_{\mathrm{e}}$, is obtained from this equation. The thermal conductivity of electron, $\kappa_{\mathrm{e}}$, is given as

$\kappa_{e}=\frac{2 k_{B}^{2} n \tau_{m e} T_{e}}{m_{e}^{*}}$

The energy of electron, $W_{\mathrm{e}}$, is given as

$W_{e}=\frac{3}{2} n k_{B} T_{e}+\frac{1}{2} n m_{e}^{*} v_{e}^{2}$

and $W_{\mathrm{e} 0}$ is the electron energy at the equilibrium state, which is given as

$W_{e 0}=\frac{3}{2} n k_{B} T_{L}$

The energy relaxation time for electrons is given by a constant value in the present calculation as $\tau_{\text {Ee-L }}=0.30$ ps. Equation (7) is the energy conservation equation for hole. The hole temperature, $T_{\mathrm{h}}$, is obtained from this equation. The thermal conductivity of hole, $\kappa_{\mathrm{h}}$, is given as

$$
\kappa_{h}=\frac{2 k_{B}^{2} p \tau_{m h} T_{h}}{m_{h}^{*}}
$$

The energy of hole, $W_{\mathrm{h}}$, is given as

$$
W_{h}=\frac{3}{2} p k_{B} T_{h}+\frac{1}{2} p m_{h}^{*} v_{h}^{2}
$$

And $W_{\mathrm{h} 0}$ is the hole energy at the equilibrium state, which is given as

$$
W_{h 0}=\frac{3}{2} p k_{B} T_{L}
$$

The energy relaxation time for holes is also given by a constant value in the present calculation as $\tau_{\text {Eh-L }}=0.30$ ps. 
Equation (8) is the energy conservation equation for crystal lattice. The non-equilibrium state in phonon system may become important for considering the deep sub-micron devices. ${ }^{(8)}$ However, prior research reported that the temperature difference between acoustic and optical phonon is relatively small ${ }^{(3)(6)}$ even if the gate length is 90 $\mathrm{nm}^{(9)}$. In this work, we focus on Si MOSFET with $90 \mathrm{~nm}$ gate length. Therefore, Fourier's law is applied in Eq. (8). The thermal conductivity of silicon is given as

$$
\kappa_{L}=154.86 \times\left(\frac{T_{L}}{300}\right)^{-\frac{4}{3}}
$$

From Eq. (8), the lattice temperature, $T_{\mathrm{L}}$, is calculated.

\section{Modeling}

Figure 1 shows the modeled Si MOSFET structure for numerical calculations. In this work, we focus on n-type Si MOSFET. The gate length, $L_{\mathrm{g}}$, is $90 \mathrm{~nm}$ and p-doped substrate with doping concentration of $N_{\mathrm{A}}=2 \times 10^{24} \mathrm{~m}^{-3}$ is considered. Each area below the source and drain electrode are highly n-doped at $N_{\mathrm{D}}=10^{25} \mathrm{~m}^{-3}$. Due to the ion doping followed by annealing process, the highly doped region is modeled to have uniform distribution of $N_{\mathrm{D}}$. The highly doping thickness, $X_{\mathrm{j}}$, is $80 \mathrm{~nm}$ and the thickness of the gate oxide, $t_{\mathrm{ox}}$, is $2 \mathrm{~nm}$. Applied bias condition is that the source electrode is grounded, and $1.0 \mathrm{~V}$ is applied to the gate and drain electrode.

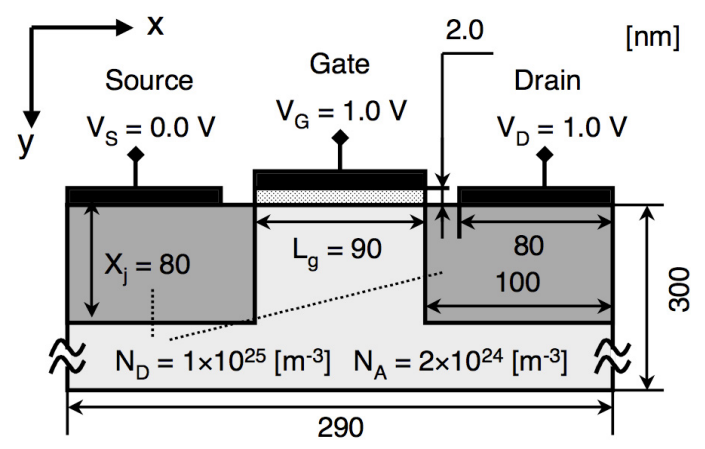

Figure 1 Modeled Device Structure

\section{Boundary Conditions}

For electrical potential, the source voltage and the drain voltage are given as constant $V_{\mathrm{S}}=0.0 \mathrm{~V}$ and $V_{\mathrm{D}}=1.0 \mathrm{~V}$. Under the gate oxide, the equation derived from Gauss's law is applied as expressed following.

$$
\left.\varepsilon_{o x} \nabla \phi\right|_{o x}=\left.\varepsilon_{S i} \nabla \phi\right|_{S i}
$$

where $\varepsilon_{\mathrm{ox}}$ means permittivity of silicon oxide. Rest of the boundary has zero gradient of potential as boundary condition.

For carrier density, constant value of $N_{\mathrm{D}}$ is given at both source and drain electrode interfaces. Zero gradient of carrier normal to the boundary is given at rest of boundary.

For carrier velocity, the velocity is zero in the perpendicular direction to the boundary except under the source and drain electrode.

For carrier temperature, constant temperature, $T_{\mathrm{e}}=T_{\mathrm{h}}=350 \mathrm{~K}$, is assumed at the bottom surface. At the source and drain electrode, it is assumed that the carrier temperature and the lattice temperature are same value. Adiabatic boundary condition is applied to the others.

For the lattice temperature, constant temperature, $T_{\mathrm{L}}=350 \mathrm{~K}$ is assumed at the bottom surface, and adiabatic boundary condition is applied to the others. 
Most of the physical properties and constants are taken from a standard literature. ${ }^{(10)}$

\section{Mesh Zoning Method for Vertical Direction}

In the electro-thermal analysis (hydrodynamic model), the continuity equation and the momentum conservation equation for electrons are below.

$$
\begin{aligned}
& \nabla \cdot\left(n \mathbf{v}_{e}\right)=-R \\
& -q n \nabla \phi+\nabla\left(n k_{B} T_{e}\right)=-\frac{n m_{e}^{*} \mathbf{v}_{e}}{\tau_{m e}}
\end{aligned}
$$

The electron current is defined as $\mathbf{J}_{e}=-q n \mathbf{v}_{e}$, and Eq. (24) can be changed. Here, only vertical (y) direction is considered.

$$
-\frac{1}{q} \frac{\partial \mathbf{J}_{e}}{\partial y}=-R
$$

By using the relationship of $\mu_{e}=q \tau_{m e} / m_{e}^{*}$, the momentum equation changes the definition of current. In the channel region of MOSFET, the electrical conductivity must be defined by the channel carrier density, $n^{*}$. Therefore, the definition of the electron current is given as

$$
\mathbf{J}_{e}=q n^{*} \mu_{e} \mathbf{E}-\mu_{e} k_{B} T_{e} \frac{\partial n}{\partial y}
$$

Setting the uniform equilibrium concentration $n_{0}$, Poisson equation can be rewritten as

$$
\frac{\partial \mathbf{E}}{\partial y}=-\frac{q\left(n-n_{0}\right)}{\varepsilon_{S i}}
$$

Substituting Eqs. (27) and (28) to Eq. (26), following equation can be obtained.

$$
\frac{q n^{*} \mu_{e}}{\varepsilon_{S i}}\left(n-n_{0}\right)-\frac{\mu_{e} k_{B}}{q} \frac{\partial^{2}\left(n T_{e}\right)}{\partial y^{2}}=-R
$$

The carrier generation/recombination term, $R$, is complex function of carrier density, $n$. Here, we don't consider this term for simplicity. So Eq. (29) becomes

$$
T_{e} \frac{\partial^{2} n}{\partial y^{2}}+2 \frac{\partial n}{\partial y} \frac{\partial T_{e}}{\partial y}+n \frac{\partial^{2} T_{e}}{\partial y^{2}}=\frac{q^{2} n^{*}}{\varepsilon_{S i} k_{B}}\left(n-n_{0}\right)
$$

From our previous results, the second and third terms at left hand side of Eq. (30) are enough small to neglect compared with other terms. ${ }^{(5)}$ Therefore the second and third term at left hand side of Eq. (30) can be negligible and Eq. (29) can be changed more simple form as shown below.

$$
\frac{\partial^{2} n}{\partial y^{2}}-\frac{q^{2} n^{*}}{\varepsilon_{S i} k_{B} T_{e}}\left(n-n_{0}\right)=0
$$

Finally, we can get the equation to be solved. Appling boundary condition, that is $n=n_{0}$ at $\mathrm{y} \rightarrow \infty$ and $n=n^{*}$ at $\mathrm{y}=0$, the solution of this equation is below.

$$
\begin{aligned}
& n=\left(n^{*}-n_{0}\right) \exp \left(-\frac{y}{L_{D}^{*}}\right)+n_{0} \\
& L_{D}^{*}=\sqrt{\frac{\varepsilon_{S i} k_{B} T_{e}}{q^{2} n^{*}}}
\end{aligned}
$$

$L_{\mathrm{D}}{ }^{*}$ means Debye length with considering the electron temperature and the local carrier density. Considering the form of Eq. (33), $L_{\mathrm{D}}{ }^{*}$ becomes larger with increasing the electron temperature. But electron temperature cannot be detected before the calculation. For accurate calculation results, smaller mesh is better. This means, if we assume the electron temperature is uniformly $350 \mathrm{~K}$, calculated $L_{\mathrm{D}}{ }^{*}$ is shorter than the actual $L_{\mathrm{D}}{ }^{*}$ and accurate MOSFET characteristics can be obtained. Therefore, $T_{\mathrm{e}}=350 \mathrm{~K}$ is assumed. $n_{\mathrm{y}=0}$ is the channel carrier density and in most case, the channel carrier density is almost similar value to the highly doped region under the source and drain electrode. This means $\mathrm{n}_{\mathrm{y}=0}^{*}=N_{\mathrm{D}}$. By 
using these equations (Eqs. (32) and (33)), the mesh zoning for the vertical direction of MOSFET can be possible as shown below.

1. Setting $n^{*}=n_{\mathrm{y}=0}{ }^{*}=N_{\mathrm{D}}, L_{\mathrm{D}}{ }^{*}$ can be obtained from Eq. (33).

2. Multiplying weight, $w$ (must be less than 1), to $L_{\mathrm{D}}{ }^{*}, \Delta \mathrm{y}_{1}$ can be obtained. This $\Delta \mathrm{y}_{1}$ is the mesh size of the first layer.

3. Substituting $\Delta \mathrm{y}_{1}$ and $n^{*}=n^{*}{ }_{\mathrm{y}=0}$ to Eq. (32), the value of $n$ at $\mathrm{y}=\Delta \mathrm{y}_{1}$ can be obtained. This $n$ becomes $n_{\mathrm{y}=\mathrm{dy} 1}^{*}$.

4. Substituting $n^{*}=n^{*}{ }_{\mathrm{y}=\mathrm{dy} 1}$ to Eq. (33), new $L_{\mathrm{D}}{ }^{*}$ can be obtained. Then multiplying $w$ to new $L_{\mathrm{D}}{ }^{*}, \Delta \mathrm{y}_{2}$ can be obtained. This $\Delta \mathrm{y}_{2}$ becomes the mesh size of the second layer.

5. Iterating $1-4$, zoned mesh can be obtained in the whole region of the calculation domain.

Appling this mesh zoning method for the vertical direction, we calculated the characteristics of MOSFET and investigated the mesh size dependence of the calculation error for the lateral direction.

\section{Accurate Mesh Size for Lateral Direction}

In following discussions, we investigate the mesh size dependence of the calculation error for the lateral direction. We used four different mesh size models. In all models, the mesh sizes for the vertical direction, $\Delta y$, are zoned by using our mesh zoning method as shown in previous section. And four different mesh sizes for the lateral direction, $\Delta \mathrm{x}$, are applied: $\Delta \mathrm{x}=1.0 \mathrm{~nm}, 2.5 \mathrm{~nm}, 5.0 \mathrm{~nm}$ and $10.0 \mathrm{~nm}$. We defined the model with $\Delta \mathrm{x}=1.0 \mathrm{~nm}$ as fine mesh model.

Table 1 shows the results of the drain current, $I_{\mathrm{D}}$, and the maximum lattice temperature, $T_{\text {Lmax }}$, of each model. In this table, $\Delta I_{\mathrm{D}}$ means the difference ratio compared to the drain current of fine mesh model and $\Delta T_{\mathrm{Lmax}}$ represents the temperature difference compared to the maximum lattice temperature of fine mesh model.

Table 1 Comparison of the Drain Current and the Maximum Lattice Temperature

\begin{tabular}{|l|l|l|l|l|}
\hline & $I_{\mathrm{D}}[\mathrm{mA} / \mathrm{mm}]$ & $\Delta I_{\mathrm{D}}[\%]$ & $T_{\mathrm{Lmax}}[\mathrm{K}]$ & $\Delta T_{\text {Lmax }}[\mathrm{K}]$ \\
\hline $1.0 \mathrm{~nm}$ & 1515 & - & 362.8 & - \\
\hline $2.5 \mathrm{~nm}$ & 1459 & 3.70 & 362.2 & 0.6 \\
\hline $5.0 \mathrm{~nm}$ & 1372 & 9.44 & 361.3 & 1.5 \\
\hline $10 \mathrm{~nm}$ & 1257 & 17.0 & 360.5 & 2.3 \\
\hline
\end{tabular}

As can be seen in this table, the results of wider mesh model show smaller value of the drain current and the maximum lattice temperature. If the case of $\Delta x=10.0 \mathrm{~nm}$ is considered, the difference ratio of the drain current compared to the drain current of fine mesh model is $17.0 \%$ and the maximum temperature difference compared to the maximum temperature of fine mesh model is $2.3 \mathrm{~K}$.

Figure 2 shows the lattice temperature, the electric field and the electron number density distributions under the gate oxide. As can been seen in Fig. 2(a), the lattice temperature is dependent on the mesh size and the results of the model with larger mesh size underestimate the lattice temperature, because the calculated drain current with larger mesh size shows lower value and the heat generation with larger mesh size is also under estimated. Further, the location of the maximum lattice temperature is also dependent on the mesh size. The location of the maximum lattice temperature shifts toward the source side with increase the size of mesh. 
Figure 2(b) shows the absolute value of electric field distributions under the gate oxide. In this graph, the maximum electric field is also underestimated with larger mesh size and the position of the maximum electric field shift to the source electrode side with increase the size of mesh. The maximum electric field of fine model is twice larger than that of $\Delta x=$ $10 \mathrm{~nm}$. In semiconductors, electrons and holes get a high energy under the high electric field and the electrons and holes transfer their energy to the crystal lattice. Thus, the different position and different magnitude of maximum electric field is strongly related to those of lattice temperature and the position of the maximum lattice temperature corresponds to the position of the maximum electric field.

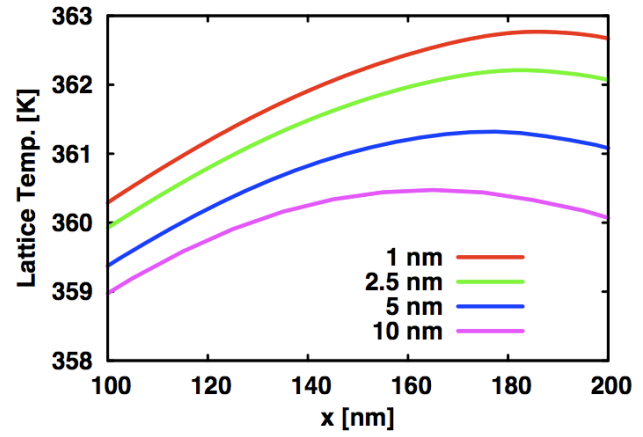

(a) Lattice Temperature

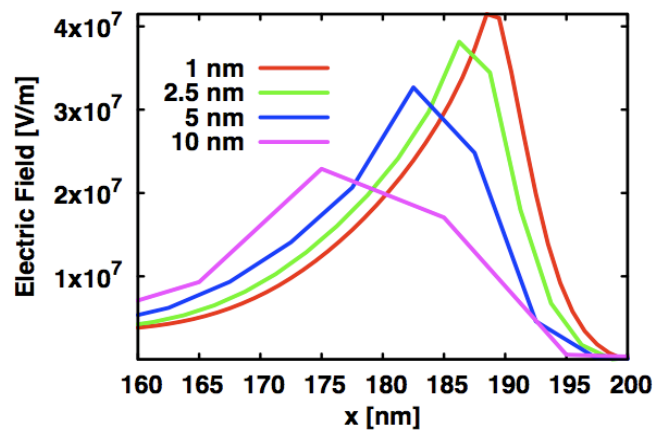

(b) Electric Field

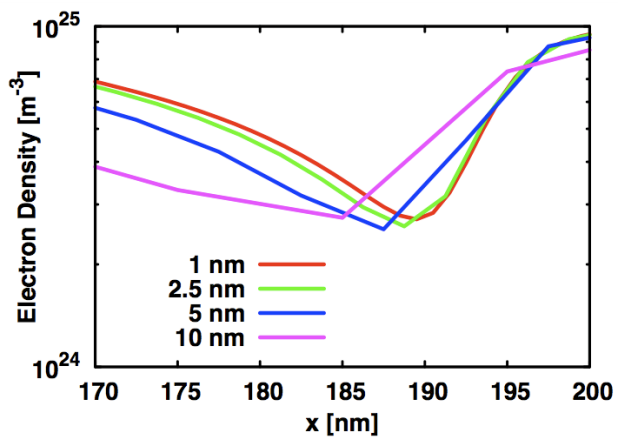

(c) Electron Density

Figure 2 Results of Lattice Temperature, Electric Field and Electron Number Density Distributions

Figure 2(c) shows the electron density underneath the gate oxide of each mesh size model. From this figure, it can be seen that the wider mesh cannot express the appropriate pinch-off point and the pinch-off point shift toward the source side if the mesh size becomes larger. In semiconductors, the carrier density is strongly related to the electrical potential. If the electron density cannot be appropriately predicted, the electrical potential distribution and the electric field become inappropriate. These results indicate that the pinch-off point should be correctly detected and the appropriate mesh has to be applied around the pinch-off point.

Figure 3 shows the electron current vectors of $\Delta x=1.0 \mathrm{~nm}$ and $2.5 \mathrm{~nm}$ model. Fig. 3(a) represents the case of $\Delta \mathrm{x}=1.0 \mathrm{~nm}$ and Fig. 3(b) represents the case of $\Delta \mathrm{x}=2.5 \mathrm{~nm}$.

In these figures, for comparing these different cases, we modified the result of the case of $\Delta \mathrm{x}=1.0 \mathrm{~nm}$. The schematic diagrams of the difference of these models and the modification procedure are shown in Fig. 4. Figure 4(a) is the mesh of $\Delta x=2.5 \mathrm{~nm}$ and Fig. 4(b) shows the mesh of $\Delta \mathrm{x}=1.0 \mathrm{~nm}$. Each result of electron current vector is located in middle of each mesh. For comparing the results of these vectors located at different positions, in the case of $\Delta \mathrm{x}=1.0 \mathrm{~nm}$ mesh, we summated the value of two and half meshes and divided the summated value by 2.5. The image of this procedure is shown in Fig. 4(c). 
As can be seen in Fig. 3, the electron current flows from the drain electrode to the source electrode through the channel region under the gate oxide. (The definition of electron current direction is opposite to the electron flow and the electron flows from the source electrode to the drain electrode.)

From Fig. 3, it can be shown that the tendencies of the electron current are quite similar. However, if each vector is separately compared, the $\mathrm{x}$ components of the electron current vector are different by more than $10 \%$ under the source electrode at the gate side edge $(x=75-80 \mathrm{~nm})$ and by more than $20 \%$ under the drain electrode at the gate side edge $(x=210-215 \mathrm{~nm})$. These differences can be seen in Fig. 5. From Figs. 3 and 5, around the edge of the source and drain electrode $(x=80 \mathrm{~nm}$ and $210 \mathrm{~nm})$, the flow direction of the electron current abruptly changes from the lateral direction to the vertical direction around the source electrode edge and from the vertical direction to the lateral direction around the drain electrode edge. If wider meshes are applied around these regions, the abrupt change of the current direction may not be able to be traced and the difference of calculated results between fine model and $\Delta \mathrm{x}=2.5 \mathrm{~nm}$ model become $10-20 \%$.

From these results, the important positions in where the mesh size for the lateral direction should be taken care are the pinch-off point, the source electrode edge at the gate side and the drain electrode edge at the gate electrode side. In following discussion, we take care of these three points and consider the possibility of mesh zoning method for the lateral direction of MOSFET.

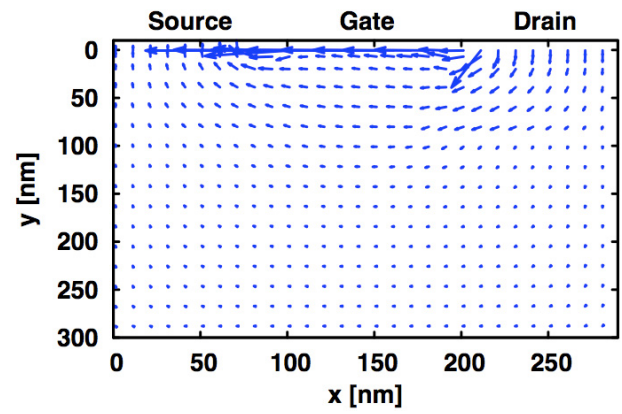

(a) $\Delta x=1.0 \mathrm{~nm}$

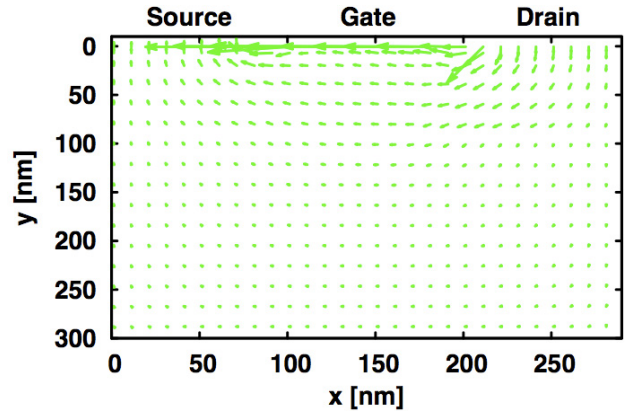

(b) $\Delta x=2.5 \mathrm{~nm}$

Figure 3 Electron Current Vectors

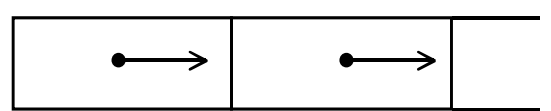

(a) $\Delta \mathrm{x}=2.5 \mathrm{~nm}$

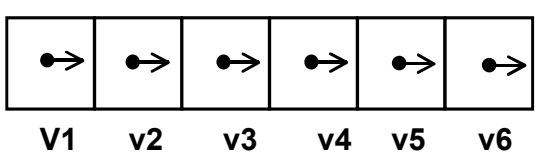

(b) $\Delta \mathrm{x}=1.0 \mathrm{~nm}$

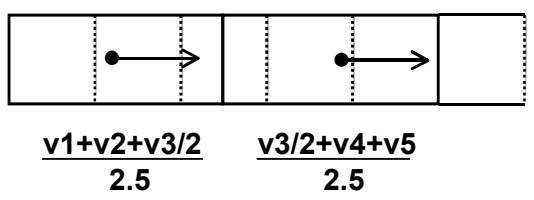

(c) $\Delta \mathrm{x}=1.0 \mathrm{~nm}$ Modified Case

Figure 4 Image of Modified Vectors 


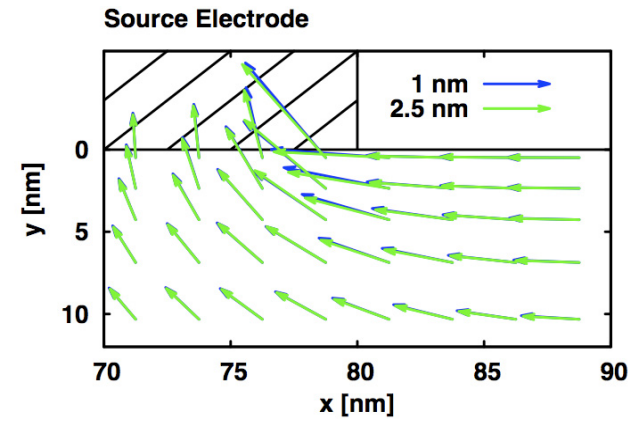

(a) Around the Source Electrode Edge

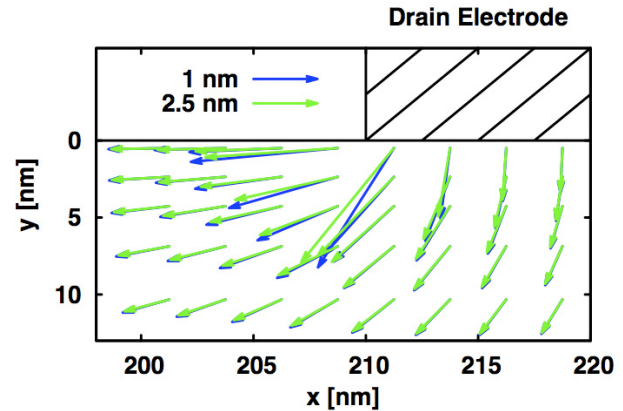

(b) Around the Drain Electrode Edge

Figure 5 Comparison of Electron Current Vectors between $1 \mathrm{~nm}$ Model and $2.5 \mathrm{~nm}$ Model

\section{Mesh Zoning Method for Lateral Direction}

In this section, we compared the results of four models to ensure the most important point in where the mesh size for the lateral direction should be given careful consideration and to consider the mesh zoning method for lateral direction of MOSFET. The details of four models are below.

Model 1 : The fine mesh model in previous calculation

Model 2: $\Delta \mathrm{x}=1.0 \mathrm{~nm}$ around the source and drain electrode edges. In other part, $\Delta \mathrm{x}=$ $2.5 \mathrm{~nm}$.

$(\Delta \mathrm{x}=1.0 \mathrm{~nm}$ mesh in $\mathrm{x}=70-90 \mathrm{~nm}$ and $\mathrm{x}=200-220 \mathrm{~nm}$. The mesh size is gradually decreased from $2.5 \mathrm{~nm}$ to $1.0 \mathrm{~nm}$ in $\mathrm{x}=60-70 \mathrm{~nm}$ and $\mathrm{x}=190-200$ $\mathrm{nm}$. The mesh size is gradually increased from $1.0 \mathrm{~nm}$ to $2.5 \mathrm{~nm}$ in $\mathrm{x}=90-100 \mathrm{~nm}$ and $\mathrm{x}=220-230 \mathrm{~nm}$. In other part, $\Delta \mathrm{x}=2.5 \mathrm{~nm}$.)

Model $3: \Delta x=1.0 \mathrm{~nm}$ around the pinch-off region. In other part, $\Delta \mathrm{x}=2.5 \mathrm{~nm}$.

$(\Delta \mathrm{x}=1.0 \mathrm{~nm}$ mesh in $\mathrm{x}=180-200 \mathrm{~nm}$. The mesh size is gradually decreased from $2.5 \mathrm{~nm}$ to $1.0 \mathrm{~nm}$ in $\mathrm{x}=170-180 \mathrm{~nm}$. The mesh size is gradually increased from $1.0 \mathrm{~nm}$ to $2.5 \mathrm{~nm}$ in $\mathrm{x}=200-210 \mathrm{~nm}$. In other part, $\Delta \mathrm{x}=2.5 \mathrm{~nm}$.)

Model 4: $\Delta \mathrm{x}=1.0 \mathrm{~nm}$ around the source and drain electrode edges and around the pinch-off region. In other part, $\Delta \mathrm{x}=2.5 \mathrm{~nm}$.

$(\Delta \mathrm{x}=1.0 \mathrm{~nm}$ mesh in $\mathrm{x}=70-90 \mathrm{~nm}$ and $180-220 \mathrm{~nm}$. The mesh size is gradually decreased from $2.5 \mathrm{~nm}$ to $1.0 \mathrm{~nm}$ in $\mathrm{x}=60-70 \mathrm{~nm}$ and $170-180 \mathrm{~nm}$. The mesh size is gradually increased from $1.0 \mathrm{~nm}$ to $2.5 \mathrm{~nm}$ in $\mathrm{x}=90-100 \mathrm{~nm}$ and $220-230 \mathrm{~nm}$. In other part, $\Delta \mathrm{x}=2.5 \mathrm{~nm}$.)

Table 2 shows the results of these models. It can be shown that the results of Model 3 and 4 show good agreement with the results of Model 1.

Figure 6 shows the lattice temperature distributions, the electric field distributions and the electron number density distributions of Model $1-4$. As can be seen in Fig. 6(a), the locations of the maximum lattice temperature of Model 3 and 4 are completely same position to that of Model 1. Figure 6(b) shows the absolute value of electric field distributions underneath the gate oxide. From this figure, the value and the position of the maximum electric field by Model 3 and 4 show good agreement with that by Model 1 . Figure 6(c) shows the electron number density distributions under the gate oxide. From Fig. 
6(c), the calculated electron number density by Model 3 and 4 also shows quite good agreement with that by Model 1.

These mean that the pinch-off region is the most important point for the lateral ( $\mathrm{x}$ ) direction in electro-thermal analysis of MOSFET. If the mesh size is taken care around the pinch-off region of MOSFET, the precise prediction of the drain current and the maximum lattice temperature can be obtained. Further, if the mesh size only around the pinch-off region is given careful consideration, the precise prediction of the location of the maximum lattice temperature and the precise prediction of the temperature distribution in the MOSFET can be obtained. Therefore, the mesh zoning method used in Model 3 is one possibility of mesh zoning for the lateral direction.

In future works, we will discuss the important length scale around the pinch-off region and the estimation procedure of the position of pinch-off point.

Table 2 Comparison of the Drain Current and the Maximum Lattice Temperature of Zoned Mesh Model

\begin{tabular}{|l|l|l|l|l|}
\hline & $I_{\mathrm{D}}[\mathrm{mA} / \mathrm{mm}]$ & $\Delta I_{\mathrm{D}}[\%]$ & $T_{\mathrm{Lmax}}[\mathrm{K}]$ & $\Delta T_{\mathrm{Lmax}}[\mathrm{K}]$ \\
\hline Model 1 & 1515 & - & 362.8 & - \\
\hline Model 2 & 1486 & 1.91 & 362.4 & 0.4 \\
\hline Model 3 & 1511 & 0.26 & 362.8 & 0.0 \\
\hline Model 4 & 1511 & 0.26 & 362.8 & 0.0 \\
\hline
\end{tabular}

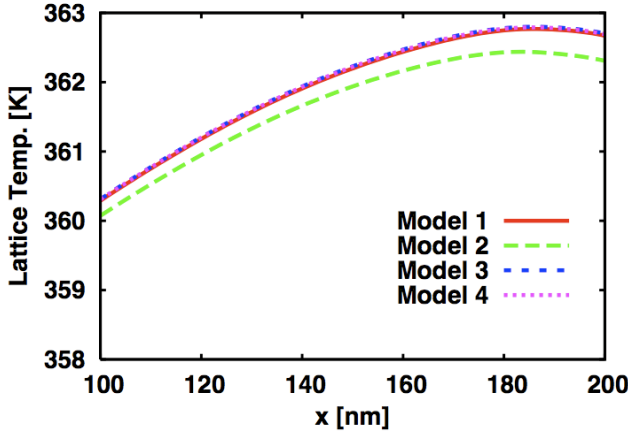

(a) Lattice Temperature

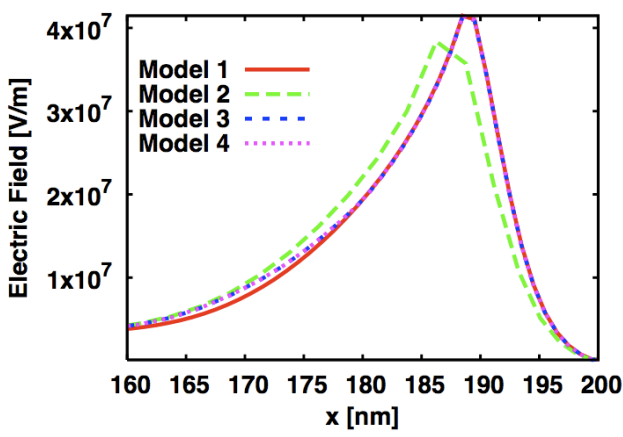

(b) Electric Field

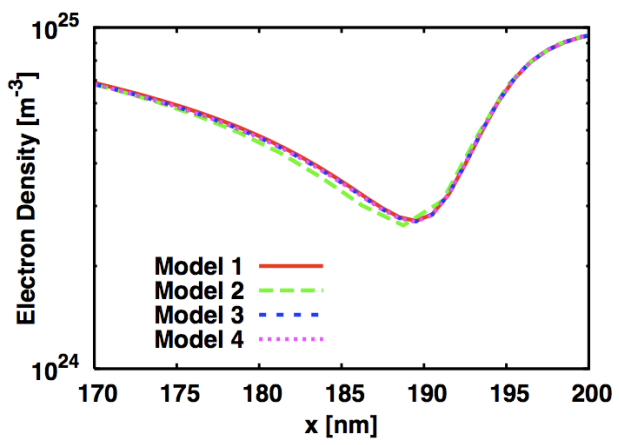

(c) Electron Density

Figure 6 Results of Lattice Temperature Distribution, Electric Field Distribution and Electron Number Density Distributions by Model 1-4

\section{Conclusion}

Electro-thermal analysis of submicron $\mathrm{Si}$ MOSFET with zoned mesh based on semiconductor physics was performed. In this research, we pointed out the most significant location in where the mesh size for the lateral direction in MOSFET should be given careful 
consideration. Calculation results showed that, if wider mesh is applied around the pn junction of drain side of MOSFET, the position of pinch-off point becomes inaccurate and the location of the maximum lattice temperature also becomes inaccurate. Further, we investigated that the most important point for taking care of the mesh size is around the pinch-off region and, if the mesh size is appropriate around the pinch-off region, the precise prediction of the drain current and the temperature distribution of MOSFET can be obtained.

In future works, we will consider the method for estimation of the position of pinch-off point and the governing length scale around the pinch-off point by using semiconductor physics theory.

\section{Acknowledgement}

The authors greatly acknowledge the support from the JSPS (Grant-in-Aid for JSPS Fellows, No. 17.8600). One of the authors (T. Hatakeyama) wishes to acknowledge JSPS for the fellowship.

\section{References}

(1) International Technology Roadmap of Semiconductors 2005 Edition, http://public.itrs.net/

(2) Fushinobu K. and Maruyama. H., Numerical Calculation of Sub-micron Hot Spot in Si Devices, Proceeding of IPACK03, (2003-7), InterPack2003-35079

(3) Tien C. L., Microscale Energy Transport, (1998), pp. 67-92 (for governing equations), pp. 264-271(for the difference between optical and acoustic phonon), Taylor \& Francis

(4) Roberts. J. W. and Chamberlain S. G., Energy-Momentum Transport Model Suitable for Small Geometry Silicon Device Simulation, COMPEL-The International Journal for Computation and Mathematics in Electrical and Electronic Engineering, Vol. 9, No. 1 (1990), pp. $1-22$

(5) Hatakeyama T., Fushinobu K. and Okazaki K., Mesh Zoning Method for Electro-Thermal Analysis of Submicron Si MOSFET, Journal of Thermal Science and Technology, Vol. 1, No. 2 (2006), pp. 101-112

(6) Lai, J. and Majumdar, A., Concurrent Thermal and Electrical Modeling of Sub-micrometer Silicon Devices, Journal of Applied Physics, Vol. 79, No. 9 (1996), pp. 7353-7361

(7) Selberherr, S., Analysis and Simulation of Semiconductor Devices, (1984), Springer-Verlag

(8) Sverdrup, P. G. Ju, Y. S., and Goodson, K. E., Sub-Continuum Simulations of Heat Conduction in Silicon-on-Insulator Transistors, Transactions of ASME Journal of Heat Transfer, Vol. 123 (2001), pp. 130-137

(9) Sinha, S. Pop, E. and Goodson, K. E., A Split-Flux Model for Phonon Transport Near Hotspots, Proceedings of IMECE04, (2004-11), IMECE2004-61949 (pp. 75-85)

(10) Sze, S. M., Physics of Semiconductor Devices, (1981) John Wiley \& Sons 